\title{
Analytic model research of sound propagation in pipe wall with sound absorption
}

\author{
Juan Ren ${ }^{1}$, Qingjun Liu $^{2, *}$, Ting Chen ${ }^{3}$, and Pingye Deng ${ }^{4}$ \\ ${ }^{1}$ Beijing Center for Physical and Chemical Analysis, Beijing, China \\ ${ }^{2}$ Beijing Academy of Science and Technology, Beijing, China \\ ${ }^{3}$ Beijing Center for Physical and Chemical Analysis, Beijing, China \\ ${ }^{4}$ Beijing Center for Physical and Chemical Analysis, Beijing, China
}

\begin{abstract}
There are a lot of principles for sound transmission in the pipeline for whether sound transmission structure or noise reduction structure. Even in ultrasonic testing, there is a large number of principles for using pipeline sound transmission. Based on the sound propagation model and the boundary conditions of pipe wall sound absorption, the sound propagation equation for pipe wall sound absorption is given by establishing mathematical model and solving mathematical equation in this paper. When the distribution of sound field along the cross-section of the pipe (outlet) is ignored, the transmission efficiency of sound with different frequencies can be calculated or the sound absorption efficiency can be calculated. The analytical solution of the sound transmission equation in the pipeline has great theoretical significance and practical value for guiding the structural design of sound transmission and noise reduction, improving the calculation efficiency and verifying the numerical analysis results.
\end{abstract}

Keywords: Sound propagation equation, Sound transmission in pipeline, Sound absorption structure.

\section{Introduction}

The propagation of sound pressure in medium satisfies the wave equation. The essence of sound is the mechanical vibration of medium, and the sound propagation is determined by wave equation. The physical properties and spatial distribution of the vibration of medium can be derived from the wave equation. For example, the wave equation of string is a special case of sound phenomenon. In the process of sound transmission, the sound pressure of different volume elements is different at the same time, and the sound pressure is different in different time in the same volume element. It can be seen that the change of sound pressure is a function of time and space. Compared with the speed of sound propagation, the measurement of sound pressure is more easy to realize, so the model with sound pressure as a function has become a widely used physical model to describe the properties of sound waves.

\footnotetext{
*Corresponding author: liuqi@,bjast.ac.cn
} 
Pipeline sound transmission is widely used in sound transmission structure and noise reduction structure [1-3]. The sound transmission process in the pipeline is characterized by relatively concentrated energy and relatively standardized waveform. The acoustic vibration of any point in the pipe is the result of the combination of incident wave and reflection wave. Thus, the sound pressure at any point in the sound propagation path is the synthesis of the incident wave sound pressure and the reflected wave sound pressure. During the process of sound propagation in the pipeline, when the section of the pipeline is transformed, the acoustic pressure and acoustic impedance are also transformed. One part of the incident wave will be reflected back to the original pipe, while the other part will continue to propagate in the new section pipeline. When the boundary effect of the pipeline is not considered, the sound propagation in the pipeline can be regarded as the plane wave propagation process.

The characteristics of pipeline sound transmission are their unique boundary conditions. Sound is transmitted along the direction of pipe diameter. When there is no sound absorption effect on the pipe wall, it can be approximately the plane propagation of sound. When there is sound absorption effect on the pipe wall, the boundary conditions of the pipe wall sound absorption are added, that is, the sound pressure of the pipe wall decreases a certain proportion compared with the sound pressure in the center of the pipe. This ratio is similar to the absorption coefficient of the wall to the sound. When sound travels along the pipeline, because it satisfies the wave equation of sound propagation, corresponding numerical analysis method can be established. At present, most of the acoustic field analysis of the pipeline structure is carried out by numerical solution. The general flow of numerical simulation is: firstly, the mathematical model is carried out for specific problems, and the appropriate control equation is found. Then, the mathematical model is discretized by the corresponding numerical method, and the computer aided operation is added to solve the problem. In engineering and scientific research, numerical methods are often used to simulate corresponding problems. Compared with traditional theoretical analysis and experimental methods, numerical simulation experiments have many advantages, such as experiments which are not convenient for observation and calculation, and can be calculated by model, with strong operability and wide application. Finite difference is a common method in numerical calculation, and they are widely used in seismic exploration and logging. Stephen [4] et al. first used finite difference method to acoustic logging problem, and transformed displacement field equation into difference equation directly. Randall [5] and others have dealt with the acoustic logging problems of various strata by using the finite difference method of velocity stress proposed by virieux [6]. Especially, the problem of all wave acoustic logging for the non axisymmetric well bore has been improved. Takemoto [7] et al. Used the finite difference time domain method to analyze the three-dimensional acoustic model accurately in 2010 and revealed the acoustic characteristics of the internal structure of the channel. In the experiment, it analyzed five Japanese vowels in detail. Wang Yuguang et al. [8] used the finite difference time domain method to simulate the two-dimensional and three-dimensional models of Mandarin Vowels in 2012, and good results were obtained. The results of these numerical analysis show that the numerical solutions of sound propagation have obtained very high precision analysis results in many cases, and play a good role in the analysis of sound transmission. However, the function and value of theoretical solution can not be replaced by numerical solution in the application of the law, trend and structure design of sound transmission.

In this paper, we use the sound pressure wave equation and the boundary condition of the sound absorption of the pipe wall to establish the mathematical model and solve the mathematical equation, and the sound transmission equation of the pipe wall is given. When ignoring the distribution of sound field along the pipe (outlet) section direction, we can calculate the transmission efficiency of different frequencies of sound through the pipe or calculate its sound absorption efficiency. The analytical solution of pipe sound transmission 
equation has great theoretical significance and practical value for guiding the structural design of sound transmission and noise reduction, improving the calculation efficiency and verifying the results of numerical analysis.

\section{The construction of sound propagation equation, sound transmission boundary condition and its solution}

\subsection{Sound propagation equation and boundary conditions of pipeline sound transmission}

Under the condition of normal temperature, normal pressure and small amplitude of sound, the propagation equation of sound in the air is as follows:

$$
\left(\frac{\partial^{2}}{\partial x^{2}}+\frac{\partial^{2}}{\partial y^{2}}+\frac{\partial^{2}}{\partial z^{2}}\right) p=\frac{1}{v^{2}} \frac{\partial^{2} p}{\partial t^{2}}
$$

where $\mathrm{p}$ is sound pressure and $v$ is sound propagation velocity. This is the free propagation equation of sound in the air.

When sound propagates along the pipe, it is absorbed or reflected by the pipe wall. According to the boundary conditions of different pipe walls, different solutions can be obtained. According to the different shape of the pipe section, the boundary conditions can be described. In general, the equation of pipe wall is as follows:

$$
f_{(x, y)}=0
$$

For example, when the pipe wall is elliptical, the equation is as follows:

$$
\frac{x^{2}}{a^{2}}+\frac{y^{2}}{b^{2}}=1
$$

Figure 1 shows the width $l x$ and height $l y$ of the pipe section when the pipe wall is of any section.

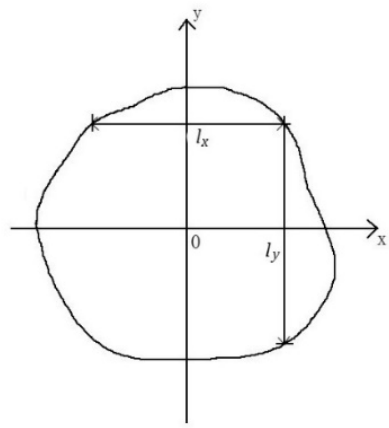

Fig. 1. Pipe wall section.

The pipe can absorb the sound pressure to a certain extent. As a qualitative analysis, in order to simplify the calculation, it is advisable to set the sound absorption coefficient of the pipe wall as $\alpha_{(\omega)}$, where $\omega$ is the angular frequency of the sound. Absorption coefficient is a function of angular frequency of sound, abbreviated as $\alpha_{\omega}$. The sound pressure in the center of the pipe is defined as $p_{c}$. Then the boundary conditions of sound transmission can be defined as:

$$
p_{(x, y, z, t)}=p_{c}\left(1-\alpha_{\omega}\right),(x, y) \in \Gamma \mid f_{(x, y)}=0
$$


Using boundary condition (4), equation (1) can be solved by trial method.

\subsection{The construction of the solution of the equation}

According to the form of equation (1), as a complex mathematical and physical equation, its solution will be a complex function. With the change of boundary conditions, the solution function will be expressed in different forms.

The input sound pressure at the initial end of the pipeline is:

$$
p_{s}=p_{0} \sin \omega t
$$

In the process of sound transmission, the sound pressure should keep its coordination characteristics. It can be concluded that:

$$
\frac{1}{v^{2}} \frac{\partial^{2} p}{\partial t^{2}}=-\frac{\omega^{2}}{v^{2}} p
$$

According to the characteristics of the wave equation, the factors matching the above factors should be included in the wave conduction of sound pressure, so that the calculation results of the second derivative in the left formula of equation (1) are consistent with those in the right formula. Referring to the characteristics of general wave function, it can be assumed that the solution of the equation contains one term:

$$
f_{1(z)}=\sin \frac{\omega z}{v}
$$

Obviously:

$$
\frac{\partial^{2} f_{1}}{\partial z^{2}}=-\frac{\omega^{2}}{v^{2}} f_{1}
$$

This factor will be equal to the derivative operation on the right side of equation (1). On this premise, equation (1) should also contain the corresponding factor, which satisfies the following homogeneous equation and its boundary conditions:

$$
\left(\frac{\partial^{2}}{\partial x^{2}}+\frac{\partial^{2}}{\partial y^{2}}+\frac{\partial^{2}}{\partial z^{2}}\right) p=0
$$

The solution of the equation must include two parts, one is the residual sound pressure waveform of the sound absorption part of the pipe wall, the other is the sound pressure waveform of the sound absorption part of the pipe wall. The two parts are separated by the weight of $1-\alpha_{\omega}$ and $\alpha_{\omega}$.

For the remaining part of the pipe wall, it can be assumed that:

$$
p_{1(x, y, z, t)}=p_{0} \sin \omega t \cdot \sin \frac{w z}{v}\left(1-\alpha_{\omega}\right)
$$

For the sound absorption part of the pipe wall, the form of the factor included in the solution can be constructed according to the homogeneous equation of equation (9) and its boundary conditions. In this case, because the influence of the remaining part of the sound absorption is separated, the boundary condition is modified as follows:

$$
p_{2(x, y, z, t)}=0,(x, y) \in \Gamma \mid f_{(x, y)}=0
$$

For the above boundary conditions, the solution of equation (9) can be constructed as follows: 


$$
\sin \frac{\pi\left(x-x_{b}\right)}{l_{x}} \sin \frac{\pi\left(y-y_{b}\right)}{l_{y}} \exp \left(-z \sqrt{\left(\frac{\pi}{l_{x}}\right)^{2}+\left(\frac{\pi}{l_{y}}\right)^{2}}\right)
$$

This solution function is only a factor in the solution of equation (1). Considering that the solution of equation (1) is composed of two parts: the sound absorbed by the pipe wall and the sound remaining after the pipe wall absorbs the sound, they should keep some same factors and can be defined without changing the conditions of the definite solution:

$$
\sin \frac{w z}{v} \alpha_{\omega}\left[\sin \frac{\pi\left(x-x_{b}\right)}{l_{x}} \sin \frac{\pi\left(y-y_{b}\right)}{l_{y}} \exp \left(-z \sqrt{\left(\frac{\pi}{l_{x}}\right)^{2}+\left(\frac{\pi}{l_{y}}\right)^{2}}\right)\right]
$$

The function is still the solution of equation (9) satisfying the boundary condition (11).

\section{Solution of sound transmission equation and pipe transmission equation}

According to the construction process of the above solution, the sound pressure solution in the hole can be assumed in the form of:

$$
\begin{array}{r}
p_{(x, y, z, t)}=p_{0} \sin \omega t \cdot \sin \frac{\omega z}{v}\left\{\left(1-\alpha_{\omega}\right)+\right. \\
\left.\alpha_{\omega}\left[\sin \frac{\pi\left(x-x_{b}\right)}{l_{x}} \sin \frac{\pi\left(y-y_{b}\right)}{l_{y}} \exp \left(-z \sqrt{\left(\frac{\pi}{l_{x}}\right)^{2}+\left(\frac{\pi}{l_{y}}\right)^{2}}\right)\right]\right\}
\end{array}
$$

where $x_{b}$ and $y_{b}$ is the starting point of $x$ and $y$ on the boundary. The above analytical solution is the characteristic solution of the equation, where $l x$ and $l y$ are regarded as discrete quantities, and their derivatives of sound pressure pair are 0 . For the convenience of calculation, a component function is defined

$$
f_{2(z)}=\exp \left(-z \sqrt{\left(\frac{\pi}{l_{x}}\right)^{2}+\left(\frac{\pi}{l_{y}}\right)^{2}}\right)
$$

These factors can be used to calculate the components of equation (1)

$$
\begin{array}{r}
\text { Right }=\frac{1}{v^{2}} \frac{\partial^{2} p}{\partial t^{2}}=-\frac{\omega^{2}}{v^{2}} p \\
\text { Left }=\left(\frac{\partial^{2}}{\partial x^{2}}+\frac{\partial^{2}}{\partial y^{2}}+\frac{\partial^{2}}{\partial z^{2}}\right) p=\left(\frac{\partial^{2}}{\partial x^{2}}+\frac{\partial^{2}}{\partial y^{2}}+\frac{\partial^{2}}{\partial z^{2}}\right)\left(p_{1}+p_{2}\right)
\end{array}
$$

Abviously:

$$
\left(\frac{\partial^{2}}{\partial x^{2}}+\frac{\partial^{2}}{\partial y^{2}}+\frac{\partial^{2}}{\partial z^{2}}\right) p_{1}=-\frac{\omega^{2}}{v^{2}} p_{1}
$$

Aimed at $p_{2}$ :

$$
\frac{\partial^{2}}{\partial x^{2}} p_{2}=-\frac{\pi^{2}}{l_{x}^{2}} p_{2}
$$




$$
\begin{array}{r}
\frac{\partial^{2}}{\partial y^{2}} p_{2}=-\frac{\pi^{2}}{l_{y}^{2}} p_{2} \\
\frac{\partial^{2}}{\partial z^{2}} p_{2}=\left(\frac{\partial^{2}}{\partial f_{1}{ }^{2}} \frac{\partial^{2} f_{1}}{\partial z^{2}}+\frac{\partial^{2}}{\partial f_{2}{ }^{2}} \frac{\partial^{2} f_{2}}{\partial z^{2}}\right) p_{2}
\end{array}
$$

The above formula can be calculated separately:

$$
\begin{array}{r}
\frac{\partial^{2}}{\partial f_{1}{ }^{2}} \frac{\partial^{2} f_{1}}{\partial z^{2}} p_{2}=-\frac{\omega^{2}}{v^{2}} p_{2} \\
\frac{\partial^{2}}{\partial f_{2}{ }^{2}} \frac{\partial^{2} f_{2}}{\partial z^{2}} p_{2}=\left[\left(\frac{\pi}{l_{x}}\right)^{2}+\left(\frac{\pi}{l_{y}}\right)^{2}\right] p_{2}
\end{array}
$$

Therefore, the left side of equation (1) is:

$$
\begin{gathered}
\left(\frac{\partial^{2}}{\partial x^{2}}+\frac{\partial^{2}}{\partial y^{2}}+\frac{\partial^{2}}{\partial z^{2}}\right)\left(p_{1}+p_{2}\right) \\
=-\frac{\omega^{2}}{v^{2}} p_{1}+\left(-\frac{\pi^{2}}{l_{x}^{2}} p_{2}\right)+\left(-\frac{\pi^{2}}{l_{y}^{2}} p_{2}\right)+\left(-\frac{\omega^{2}}{v^{2}} p_{2}\right)+\left[\left(\frac{\pi}{l_{x}}\right)^{2}+\left(\frac{\pi}{l_{y}}\right)^{2}\right] p_{2} \\
=-\frac{\omega^{2}}{v^{2}}\left(p_{1}+p_{2}\right)+\left(-\frac{\pi^{2}}{l_{x}^{2}} p_{2}\right)+\left(-\frac{\pi^{2}}{l_{y}^{2}} p_{2}\right)+\left[\left(\frac{\pi}{l_{x}}\right)^{2}+\left(\frac{\pi}{l_{y}}\right)^{2}\right] p_{2} \\
=-\frac{\omega^{2}}{v^{2}}\left(p_{1}+p_{2}\right)=-\frac{\omega^{2}}{v^{2}} p
\end{gathered}
$$

Compared with equation (11), the analytical solution of equation (6) can satisfy equation (1), and the left and right sides of the equation are equal.

According to $\sin \frac{\pi\left(x-x_{b}\right)}{l_{x}} \sin \frac{\pi\left(y-y_{b}\right)}{l_{y}}$, when $(x, y) \in \Gamma$, the value of sine function is 0 , which leads to $p_{2}=0$. The sound pressure at the boundary is:

$$
p_{1(x, y, z, t)}=p_{0} \sin \omega t \cdot \sin \frac{w z}{v}\left(1-\alpha_{\omega}\right)=p_{\mathrm{c}}\left(1-\alpha_{\omega}\right)
$$

It can be seen that the analytical solution of equation (6) satisfies the boundary conditions defined in equation (4).

\section{Deduction and application of pipe sound transmission equation}

In general, the analysis of sound field does not pay attention to the distribution of sound field along the cross section of the pipe:

$$
p_{(z, t)}=p_{0} \sin \omega t \cdot \sin \frac{w z}{v}\left\{\left(1-\alpha_{\omega}\right)+\alpha_{\omega} \exp \left(-z \sqrt{\left(\frac{\pi}{l_{x}}\right)^{2}+\left(\frac{\pi}{l_{y}}\right)^{2}}\right)\right\}
$$

where $l x$ and $l y$ are the width and height of the pipe section.

The distribution of sound pressure fluctuation amplitude along the pipe diameter direction is as follows: 


$$
p_{(z)}=p_{0}\left\{\left(1-\alpha_{\omega}\right)+\alpha_{\omega} \exp \left(-z \sqrt{\left(\frac{\pi}{l_{x}}\right)^{2}+\left(\frac{\pi}{l_{y}}\right)^{2}}\right)\right\}
$$

When the absorption coefficient of sound pressure is 1 , the distribution of sound pressure fluctuation amplitude along the pipe diameter direction is further simplified as follows:

$$
p_{(z)}=p_{0} \exp \left(-z \sqrt{\left(\frac{\pi}{l_{x}}\right)^{2}+\left(\frac{\pi}{l_{y}}\right)^{2}}\right)
$$

In this simplified case, the ratio of the amplitude of sound pressure fluctuation to the amplitude of input sound pressure is as follows:

$$
p_{(z)} / p_{0}=\exp \left(-z \sqrt{\left(\frac{\pi}{l_{x}}\right)^{2}+\left(\frac{\pi}{l_{y}}\right)^{2}}\right)
$$

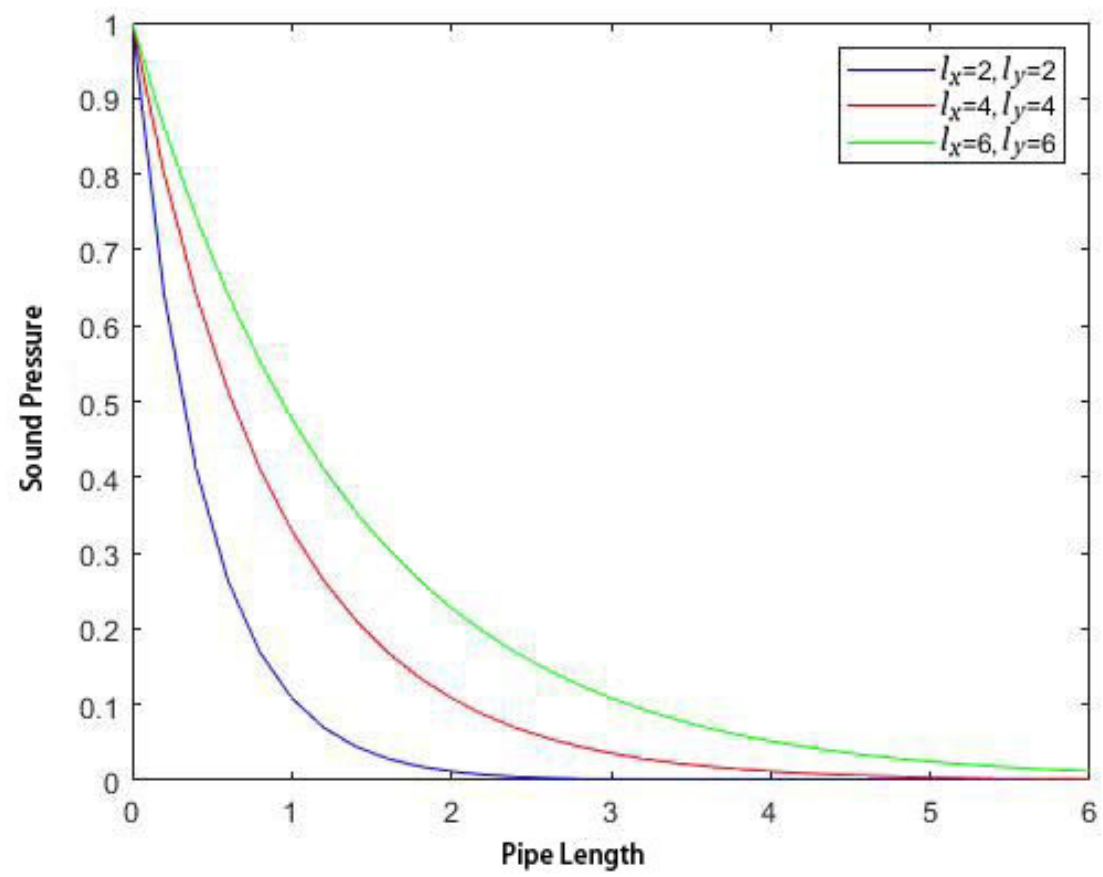

Fig. 2. Relationship between sound pressure and tube length at $p_{0}=1$.

Figure 2 shows when $p_{0}=1$, the relationship between sound pressure and tube length. It can be seen that the sound pressure decreases exponentially along the pipe diameter when the sound propagates in the sound-absorbing pipe. The decreasing exponent is directly proportional to the length of the pipe and inversely proportional to the section size of the pipe.

When $\mathrm{z}=l x=l y=r$, the ratio of the output amplitude to the input amplitude is about:

$$
\frac{p_{\text {out }}}{p_{\text {in }}}=\exp (-\pi \sqrt{2}) \approx 0.1
$$


Therefore, when the sound absorption efficiency of the pipe wall is high (nearly 100\%), the amplitude of the output sound pressure will reach about 0.1 as long as the pipe length reaches the same level as its cross-section size. This conclusion provides a theoretical basis for the sound absorption of pipes.

Meanwhile, in the scene where voice and music need to be shaped, some sound that needs to be removed can be effectively suppressed by pipeline transmission, and an effective voice transmission structure can be constructed for the sound to be clean and tidy. This feature can also be used to load useful information for sound. For example, voice is to adjust the length of $l x$ and $l y$ and speech channel (pipe) through the change of mouth type to suppress some speech frequencies, so as to send out different voice and represent different information.

\section{Conclusion}

The sound propagation in the pipeline conforms to the general law of sound propagation in the air, that is, it satisfies the wave equation of sound pressure. When sound propagates in the pipe with sound absorption on the pipe wall, the boundary conditions of sound absorption on the pipe wall should also be satisfied. Based on the wave equation of sound propagation, this paper analyzes the analytical solution of sound propagation along the pipe when the pipe wall absorbs sound. Through the calculation of the related equations and formulas, it is proved that the analytical solution equation recommended in this paper satisfies the sound pressure wave equation of sound propagation in the air, and satisfies the sound transmission boundary condition of pipe wall sound absorption. Therefore, the equation is the analytical solution of the pipe sound transmission equation with sound absorption on the pipe wall.

The analytical solution recommended in this paper shows that the absorption coefficient of the pipe wall to the sound pressure is a key parameter. When the absorption coefficient of the pipe wall to the sound pressure is less than $100 \%$, for example, a part of the sound pressure at the input end propagates to the output end through the pipe. When the absorption coefficient of the pipe wall to the sound pressure is close to $100 \%$, the sound absorption efficiency of the pipe can be very high. When the length of the pipe is equal to its crosssection size, the sound absorption effect of the pipe is close to $99 \%$.

In the process of pipe sound transmission, the overall absorption effect of sound is inversely proportional to the cross-section size of the pipe and directly proportional to the length of the pipe. This feature can be used in the design of sound absorption structure, also can be used in the design of musical instrument structure which needs certain filtering function, and can also be used in the frequency analysis of speech in the process of transmission.

\section{Reference}

1. Sun Zhongzheng. Transmission Loss Test of Duct Wall Sound Pressure Level [J]. Noise and Vibration Control. 2019, 5, Vol. 39: 250-254.

2. Hou Wei, Pan Haoran, Song Weihua, et al. Numerical simulation of nonlinear propagation of sound waves in a finite horn [J]. Acta Acustica. 2015, 4, Vol. 40, P569578.

3. Liu Cui Wei, Li Yuxing, Wang Wuchang, et al. Theoretical study and experimental study on leak detection for natural gas pipelines based on acoustic method [J]. Acta Acustica. 2013, 3, Vol. 38, P372-381.

4. Stephen R A, Pardo-Casas F and Cheng C H. Finite-difference Synthetic acoustic logs[J]. Geophysics, 1985, 50: 1588-1609. 
5. Randall C J . Muitipole acoustic Waveforms in Nonaxisymmetric Boreholes and Formations[J]. J. Acoust. Soc. Am., 1991, 90(3): 1620-1631.

6. Vixieux J, SH-wave Propagation in Heterogeneous Media: Velocity-stress Finitedifference Method[J], Geophysics 1984, 49(11): 1933-1942.

7. Takemoto H, Mokhtari P, Kitamura T. Acoustic analysis of the vocal tract during vowel production by finite - difference time - domain method[J]. Journal of the Acoustical Society of America, 2010, 128 (6): 3724.

8. Wang Y, Wang H, Wei J, et al. Mandarin vowel synthesis based on 2D and 3D vocal tract model by finite-difference time-domain method[C]. In Signal and Information Processing Association Summit and Conference, 2012: 1-4. 